\title{
MEASURE EXPANSIVE AND DOMINATED SPLITTING
}

\author{
DAEJUNG KiM*
}

\begin{abstract}
In this paper, we show that if a nontrivial transitive set is $C^{1}$-stably measure expansive, then it admits a dominated splitting.
\end{abstract}

\section{Introduction}

Let $M$ a compact connected $C^{\infty}$ Riemannian manifold without boundary, and $\operatorname{Diff}^{1}(\mathrm{M})$ be the space of diffeomorphisms of $M$ endowed with the $C^{1}$-topology. Denote by $d$ the distance on $M$ induced from the Riemannian metric $\|\cdot\|$ on the tangent bundle $T M$. Let $f \in \operatorname{Diff}^{1}(\mathrm{M})$.

The notion of expansiveness was introduced by Utz [8] in the middle of the twentieth century. Roughly speaking, a system is expansive if two orbits cannot remain close to each other under the action of the system. This notion is very important in the context of the theory of dynamical systems, for example, the proof of the existence of Markov partitions. As pointed out by Morales [6], in light of the rich consequences of expansiveness in the dynamics of a system, it is natural to consider another notions of expansiveness.

Let us start with the different definitions of expansiveness we shall deal with. Given $x \in M$ and $\delta>0$, define the dynamical $\delta$-ball, the set

$$
\Gamma_{\delta}(x, f)=\left\{y \in M: d\left(f^{i}(x), f^{i}(y)\right) \leq \delta, i \in \mathbb{Z}\right\} .
$$

We simply write $\Gamma_{\delta}(x)$ instead of $\Gamma_{\delta}(x, f)$ when it is understood which we refer to.

A diffeomorphism $f: M \rightarrow M$ is called expansive if there is $\delta>0$ such that for every pair of different points $x, y \in M$ there is $n \in \mathbb{Z}$ such that $d\left(f^{n}(x), f^{n}(y)\right)>\delta$. Equivalently, $f$ is expansive if there is $\delta>0$ such that $\Gamma_{\delta}(x)=\{x\}$ for all $x \in M$.

Received September 27, 2013; Accepted October 11, 2013.

2010 Mathematics Subject Classification: Primary 37D30.

Key words and phrases: expansive, measure expansive, dominated splitting. 
To describe our result, we give some notations on probability measures of $M$. Denote by $\mathcal{M}(M)$ the set of Borel probability measures of $M$ endowed with the weak topology, and $\mathcal{M}_{f}(M) \subset \mathcal{M}(M)$ the set of $f$-invariant measures. We say that $\mu \in \mathcal{M}(M)$ is non-atomic if it satisfies $\mu(\{x\})=0$ for each $x \in M$. It is known that the set of non-atomic measures is a residual set in $\mathcal{M}(M)$ (See [3]).

We introduce the notion of measure expansiveness given by Morales. Let $\mu$ be a Borel probability measure which is not necessarily $f$-invariant. We say that $f$ is measure expansive (or $\mu$-expansive) if there is $\alpha>0$ such that $\mu\left(\Gamma_{\alpha}(x)\right)=0$ for all $x \in X$. Note that if $f$ is expansive then $f$ is $\mu$-expansive. But the converse is not true (See [6]). In [6], the basic property of measure-expansive homeomorphisms was studied, and different proofs of the famous results for expansive homeomorphisms were given from a view point of measure theory, for instance, the nonexistence of expansive homeomorphisms on the circle.

Let $\Lambda$ be a closed $f$-invariant set. We say that $\Lambda$ is locally maximal if there is a neighborhood $U$ of $\Lambda$ such that $\Lambda=\bigcap_{n \in \mathbb{Z}} f^{n}(U)$.

Definition 1.1. Let $\Lambda$ be a closed invariant set of $f \in \operatorname{Diff}^{1}(\mathrm{M})$. We say that $f$ is $C^{1}$-stably $\mu$-expansive if there are a compact neighborhood $U$ of $\Lambda$ and a $C^{1}$-neighborhood $\mathcal{U}(f)$ of $f$ such that $\Lambda=\Lambda_{f}(U)=$ $\bigcap_{n \in \mathbb{Z}} f^{n}(U)$, and for any $g \in \mathcal{U}(f), \Lambda_{g}(U)$ is $\mu$-expansive, where $\Lambda_{g}(U)=$ $\bigcap_{n \in \mathbb{Z}} g^{n}(U)$ is the continuation of $\Lambda$.

Recall that a compact invariant set $\Lambda$ is called transitive if $\omega(x)=\Lambda$ for some $x \in \Lambda$.

Recall the notion of a dominated splitting for a compact $f$-invariant subset $\Lambda \subset M$ of a diffeomorphism $f: M \rightarrow M$. It can be seen as a weak form of hyperbolicity. But dominated splitting is also a robust property and it is an important mechanism for many dynamical phenomena.

We say that a compact $f$-invariant set $\Lambda \subset M$ admits a dominated splitting if the tangent bundle $T_{\Lambda} M$ has a continuous $D f$-invariant splitting $E \oplus F$ and there exist $C>0,0<\lambda<1$, such that

$$
\left\|D f^{n}\left|E(x)\|\cdot\| D f^{-n}\right| F\left(f^{n}(x)\right)\right\| \leq C \lambda^{n} \quad \text { for all } \quad x \in \Lambda, n \geq 0 .
$$

In this paper, we study measure expansiveness and dominated splitting. In differentiable dynamical systems, dominated splitting is a natural generalization of hyperbolicity. We say that $\Lambda$ is nontrivial if $\Lambda$ is not one orbit. The following is the main result in this paper.

TheOREM 1.2. Let $\Lambda$ be a nontrivial transitive set of $f \in \operatorname{Diff}^{1}(\mathrm{M})$. If $\Lambda$ is $C^{1}$-stably $\mu$-expansive, then it admits a dominated splitting. 


\section{Proof of Theorem 1.2}

Let $M$ be as before, and let $f \in \operatorname{Diff}^{1}(\mathrm{M})$. In this section, we introduce the notation of pre-sink (resp. pre-source). A periodic point $p$ of $f$ is called a pre-sink (resp. pre-source) if $D f^{(p)}(p)$ has a multiplicity one eigenvalue with modulus 1 and the other eigenvalues has norm strictly less than 1 (resp. bigger than 1).

The metric between compact sets is the Hausdorff metric. We use $d_{H}$ to denote the distance of the Hausdorff distance. The limits of compact sets are under the Hausdorff distance. The following lemma is on the limit of uniformly dominated splitting.

Lemma 2.1. [1, Lemma 1.4] Let $g_{n}$ converges to $f$, and $\Lambda_{n}$ be a closed $g_{n}$-invariant set such that the Hausdorff limit of $\Lambda_{n}$ equal to $\Lambda$. If $\Lambda_{g_{n}}(U)$ admits a $l$-dominated splitting respecting $g_{n}$, then $\Lambda$ admits an $l$-dominated splitting respecting $f$.

To prove Theorem 1.2, it is enough to show the following proposition.

Proposition 2.2. Let $\Lambda$ be a nontrivial transitive set of $f$. If $\Lambda$ is $C^{1}$-stably $\mu$-expansive for $f$, and there exist a sequence $g_{n}$ goes to $f$ and periodic orbits $P_{n}$ of $g_{n}$ which converges to $\Lambda$ in Hausdorff limits, then $\Lambda$ admits a dominated splitting.

To prove Proposition 2.2, it is enough to show that the following lemma.

Lemma 2.3. Let $\Lambda$ be a nontrivial transitive set of $f$. Suppose that $\Lambda$ is $C^{1}$-stably $\mu$-expansive for $f$. Let $U$ and $U(f)$ be given in the Definition 1.1. Then for any $g \in \mathcal{U}(f), g$ has neither pre-sink nor pre-sources with the orbit staying in $U$.

Proof. To induce the contradiction, we assume that there is $g \in U(f)$ such that $g$ has a pre-sink $p$ with $\operatorname{Orb}(p) \subset U$. By the well-known Franks Lemma, we can linearize $\mathrm{g}$ at $\mathrm{p}$ with respect to the exponential coordinates $\exp _{p}$, i.e, after an arbitrarily small perturbation, we can get a diffeomorphism $g_{1} \in U(f)$ such that there is $\epsilon_{1}>0$ small enough with $B_{\epsilon_{1}}(\operatorname{Orb}(p)) \subset U$ such that

$$
\left.g_{1}\right|_{B_{\epsilon_{1}}\left(g^{i}(p)\right)}=\left.\exp _{g^{i+1}(p)} \circ D_{g^{i}(p)} g \circ \exp _{g^{i}(p)}^{-1}\right|_{B_{\epsilon_{1}}\left(g^{i}(p)\right)},
$$

for any $0 \leq i \leq \pi(p)-1$.

Since $p$ is pre-sink of $g, D_{p} g^{\pi(p)}$ has a multiplicity one eigenvalue such that $|\lambda|=1$ and other eigenvalues of $D_{p} g^{\pi(p)}$ have moduli less 
than 1 . Denote by $E_{p}^{c}$ the eigenspace corresponding to $\lambda$, and $E_{p}^{s}$ the eigenspace corresponding to the eigenvalues with modulus less than 1 . Thus $T_{p} M=E_{p}^{c} \oplus E_{p}^{s}$. If $\lambda \in \mathbb{R}$, then $\operatorname{dim} E_{p}^{c}=1$, and if $\lambda \in \mathbb{C}$, then $\operatorname{dim} E_{p}^{c}=2$.

Let $\mathfrak{M}_{\mathcal{I}_{p}}$ be the normalized Lebesgue measure on $\mathcal{I}_{p}$. Define $\mu \in$ $\mathcal{M}_{f}(M)$ by

$$
\mu(c)=\frac{1}{\pi(p)} \sum_{j=0}^{\pi(p)-1} \mathfrak{M}_{\mathcal{I}_{p}}\left[f^{-j}\left(C \cap f^{j}\left(\mathcal{I}_{p}\right)\right)\right]
$$

for any Borel sets $C$ of $M$. It is clear that $\mu$ is a non-atomic measure. At first, we consider the case $\operatorname{dim} E_{p}^{c}=1$. For simplicity, we suppose that $\lambda=1$, and $g_{1}^{\pi(p)}(p)=p$. The case of $\lambda=-1$ can be proved similarly. Since the eigenvalue $\lambda=1$, there is a small $\operatorname{arc} \mathcal{I}_{p} \subset B_{\epsilon_{1}}(p) \cap \exp _{p}\left(E_{p}^{c}\left(\epsilon_{1}\right)\right)$ centered at $p$ such that $\left.g_{1}^{\pi(p)}\right|_{\mathcal{I}_{p}}$ is the identity map. Here $E_{p}^{c}\left(\epsilon_{1}\right)$ is the $\epsilon_{1}$-ball in $E_{p}^{c}$ center at the origin $O_{p}$. Since $\left.g_{1}^{\pi(p)}\right|_{\mathcal{I}_{p}}$ is the identity map, we know that $g_{1}^{\pi(p)} \mid \mathcal{I}_{p}$ is not $\mu$-expansive. That is, Since $\left.g_{1}^{\pi(p)}\right|_{\mathcal{I}_{p}}=i d$,

$$
\left\{y \in \mathcal{I}_{p}: d(p, y)<\delta_{1}\right\} \subset \Gamma_{\delta}(p) .
$$

Thus, we have

$$
\mu\left(\Gamma_{\delta}(p)\right) \geq \mu\left(\left\{y \in \mathcal{I}_{p}: d(p, y)<\delta_{1}\right\}\right)>0 .
$$

This is a contradiction.

Finally, we consider the case $\operatorname{dim} E_{p}^{c}=2$. In this proof, to avoid the notational complexity, we may assume that $g(p)=p$. As in the first case, by Franks Lemma, there are $\epsilon_{1}>0$ and $g_{1} \in \mathcal{U}(f)$ such that $g_{1}(p)=g(p)=p$ and

$$
g_{1}(x)=\exp _{p} \circ D_{p} g \circ \exp _{p}^{-1}(x)
$$

if $x \in B_{\epsilon_{1}}(p)$. With a $C^{1}$-small modification of the map $D_{p} g$, we suppose that there is $l>0$ such that $D_{p} g^{l}(v)=v$ for any $v \in E_{p}^{c}\left(\epsilon_{1}\right) \cap$ $\exp _{p}^{-1}\left(B_{\epsilon}(p)\right)$. By our assumption, $\left.g_{1}\right|_{\exp _{p}\left(E_{p}^{s}\left(\epsilon_{1}\right)\right) \cap B_{\epsilon_{1}}(p)}$ of the map is contraction. Take $v \in E_{p}^{c}\left(\epsilon_{1}\right)$ such that $\|v\|=\frac{\epsilon_{1}}{4}$, and set

$$
\mathcal{C}_{p}=\exp _{p}\left(\left\{t \cdot v: 1 \leq t \leq 1+\frac{\epsilon_{1}}{4}\right\}\right) \cap B_{\epsilon_{1}}(p) .
$$

Then $g_{1}^{l}\left(\mathcal{C}_{p}\right)=\mathcal{C}_{p}$ and $\left.g_{1}^{l}\right|_{\mathcal{C}_{p}}$ is the identity map. Then by similar arguments as above, we get the contradiction. 
Let $G L(n)$ be the group of linear isomorphisms of $\mathbb{R}^{n}$. A sequence $\xi: \mathbb{Z} \rightarrow G L(n)$ is called periodic if there is $k>0$ such that $\xi_{j+k}=\xi_{j}$ for $k \in \mathbb{Z}$. We call a finite subset $A=\left\{\xi_{i}: 0 \leq i \leq k-1\right\} \subset G L(n)$ is a periodic family with period $k$. For a periodic family $A=\left\{\xi_{i}: 0 \leq i \leq\right.$ $n-1\}$, we denote $\mathcal{C}_{\mathcal{A}}=\xi_{n-1} \circ \xi_{n-2} \circ \cdots \xi_{0}$.

We consider about uniformly contracting family. Let $A=\left\{\xi_{i}: 0 \leq\right.$ $i \leq k-1\} \subset G L(n)$ be a periodic family. We say the sequence $A$ is uniformly contracting family if there is a constant $\delta>0$ such that any $\delta$-perturbation of $A$ are sink, i.e., for any $B=\left\{\zeta_{i}: 0 \leq i \leq k-1\right\}$ with $\left\|\zeta_{i}-\xi_{i}\right\|<\delta$, all eigenvalue of $\mathcal{C}_{\mathcal{B}}$ have moduli less than 1 . Similarly, we can define the uniformly expanding periodic family.

The following lemma is well known result. In fact, we make using the $C^{1}$-closing lemma and property of transitive set. Hereafter, we consider nontrivial transitive set.

Lemma 2.4. [9, Corrollary 2.7.1] Let $\Lambda$ be a transitive set. Then there are a sequence $\left\{g_{n}\right\}$ of diffeomorphism and a sequence $\left\{P_{n}\right\}$ of periodic orbits of $g_{n}$ with period $\pi\left(P_{n}\right) \rightarrow \infty$ such that $g_{n} \rightarrow f$ in the $C^{1}$-topology and $P_{n} \rightarrow_{H} \Lambda$ as $n \rightarrow \infty$, where $\rightarrow_{H}$ is the Hausdorff limit, and $\pi\left(P_{n}\right)$ is the period of $P_{n}$.

Let $P_{n}$ be a periodic orbit sequence of $f$. Choose $p_{n} \in P_{n}$, then we get a linear map sequence $A_{n}=\left\{D_{p_{n}} f, D_{f\left(p_{n}\right)} f, \cdots, D_{f^{\pi\left(p_{n}\right)-1}\left(p_{n}\right)} f\right\}$.

Lemma 2.5. [4, Lemma 3.2] If $\Lambda$ is not a periodic orbit and $A_{n}$ is given in the above. Then for any $\epsilon>0$ there exists an $n_{0}(\epsilon)>0$ such that for any $n>n_{0}(\epsilon), A_{n}$ is neither $\epsilon$-uniformly contracting nor $\epsilon$-uniformly expanding.

From the above lemmas and main conclusion of [2], one can get the following lemma.

Lemma 2.6. [4, Lemma 3.3] Let $g_{n}$ and $P_{n}$ be given as in the assumption of Proposition 2.2. Then for any $\epsilon>0$ there are $n(\epsilon), l(\epsilon)>0$ such that for any $n>n(\epsilon)$ if $P_{n}$ does not admit an $l(\epsilon)$ dominated splitting, then one can find $g_{n}^{\prime} C^{1} \epsilon$-close $g_{n}$ and preserving the orbit of $P_{n}$ such that $P_{n}$ is pre-sink or pre-source respecting $g_{n}^{\prime}$.

From the above lemmas and Lemma 2.1, we can get Proposition 2.2.

End of proof of Theorem 1.2 Let $\Lambda$ be a nontrivial transitive set of $f \in \operatorname{Diff}^{1}(\mathrm{M})$. Then by Lemma 2.4, there exist a sequence $\left\{g_{n}\right\}$ of diffeomorphism and a periodic orbit $P_{n}$ of $g_{n}$ such that $g_{n} \rightarrow f$ in the $C^{1}$ topology and $P_{n} \rightarrow \Lambda$ in the Hausdorff limit. By Lemma 2.6, $P_{n}$ admits 
a $l$-dominated splitting. Thus by Lemma $2.1, \Lambda$ admits a $l$-dominated splitting.

\section{References}

[1] C. Bonatti, L. J. Diaz, and E. A. Pujals, $C^{1}$-generic dichotomy for diffeomorphisms: weak forms of hyperbolicity or infinitely many sinks or sources, Ann. of Math. 158 (2003), 187-222.

[2] C. Bonatti, N. Gourmelon, and T. Vivier, Perturbations of the derivative along periodic orbits, Ergodi. Th. \& Dynm. Syst 26 (2006), 1307-1337.

[3] M. Denker, C. Grillenberger, and K. Sigmund, Ergodic Theory on Compact Spaces, Lecture Notes in Math. 527, Springer-Verlag, Berlin, 1976.

[4] K. Lee, G. Lu, and X. Wen, $C^{1}$-stably weak shadowing property of chain transitive sets, preprint.

[5] M. Lee, Continuum-wise expansive and dominated splitting, Int. Jour. of Math. Analysis, Vol. 7 (2013), no. 23, 1149-1154.

[6] C. A. Morales, Measure expansive systems, Preprint IMPA, D083, 2011.

[7] M. J. Pacifico and J. L. Vieitez, On measure expansive diffeomorphisms, arXiv: $1302.2282 \mathrm{v} 1$.

[8] W. R. Utz, Unstable homeomorphisms, Proc. Amer. Math. Soc. 1 (1950), 769774 .

[9] D. Yang, Stably weakly shadowing transitive sets and dominated splitting, Proc. Amer. Math. Soc. 139 (2011), 2747-2751.

Daejeon Science High School

Daejeon 305-338, Republic of Korea

E-mail: kimdj623@hanmail.net 